08.2

\title{
Асимптотическая стадия роста автокаталитических III-V нитевидных нанокристаллов методом молекулярно-пучковой эпитаксии
}

\author{
() В.Г. Дубровский ${ }^{1}$, М.В. Рылькова ${ }^{1}$, А.С. Соколовский ${ }^{1}$, Ж.В. Соколова ${ }^{1,2}$ \\ ${ }^{1}$ Санкт-Петербургский государственный университет, Санкт-Петербург, Россия \\ ${ }^{2}$ Санкт-Петербургский государственный экономический университет, Санкт-Петербург, Россия \\ E-mail: dubrovskii@mail.ioffe.ru
}

Поступило в Редакцию 11 октября 2021 г.

В окончательной редакции 11 октября 2021 г.

Принято к публикации 18 октября 2021 г.

\begin{abstract}
Впервые построена аналитическая теория автокаталитического роста III-V нитевидных нанокристаллов (ННК) методом молекулярно-пучковой эпитаксии на асимптотической стадии, когда ННК адсорбируют все атомы III группы, поступающие из газовой фазы. Найдено выражение для длины затенения, соответствующее полной блокировке потока на поверхность подложки при молекулярно-пучковой эпитаксии. Получены решения для длины и радиуса ННК в зависимости от толщины осажденного материала. Показано, что длина ННК увеличивается, а их радиус уменьшается при уменьшении расстояния между ННК и увеличении соотношения потоков V/III.
\end{abstract}

Ключевые слова: III-V нитевидные нанокристаллы, эффект затенения, длина, радиус, поверхностная плотность, моделирование.

DOI: 10.21883/PJTF.2022.03.51976.19049

III-V нитевидные нанокристаллы (ННК) являются перспективными объектами для создания оптоэлектронных гетероструктур, совмещенных с кремниевой платформой [1]. Благодаря эффективной релаксации упругих напряжений на боковых поверхностях III-V HНК могут выращиваться на рассогласованных подложках кремния без формирования дислокаций несоответствия [2,3]. Например, известные сложности получения когерентного InAs на Si (рассогласование решеток 11.6\%) [4] могут быть преодолены в геометрии ННК с диаметром менее критического (25 nm) [3]. III-V ННК выращиваются по механизму пар-жидкость-кристалл (ПЖК) с использованием катализаторов $\mathrm{Au}$ [5] или металлов III группы (Ga, In) (в последнем случае ПЖК-рост называют автокаталитическим [6]) либо методом селективной эпитаксии [7]. Большинство используемых моделей роста ННК (их обзор можно найти в [8]) относится к одиночному ННК и пренебрегает коллективными эффектами, в том числе эффектом затенения (или теневым эффектом) блокировки направленных потоков на подложку и боковые поверхности ННК при молекулярно-пучковой эпитаксии (МПЭ) [9].

Поток атомов группы III (Ga), попадающий на поверхность подложки между ННК, приводит либо к росту паразитного слоя $[10,11]$, либо к отражению от поверхности оксидной маски [12]. Важную роль в кинетике роста ННК играет их радиальное расширение [11], особенно для автокаталитических ННК [13]. В работе [13] дан теоретический анализ данных по МПЭ-росту автокаталитических НHК GaP в упорядоченных массивах отверстий на поверхностях $\mathrm{SiO}_{x} / \mathrm{Si}(111)$ с учетом отраженного потока $\mathrm{Ga}$, радиального роста и эффекта затенения подложки. Было показано, что поток $\mathrm{Ga}$ на ННК стремится к максимальному значению, равному прямому потоку на площадь поверхности, приходящейся на один ННК. Целью настоящей работы является построение теории МПЭ-роста ННК на этой стадии.

Рассматриваются потоки элементов групп III (индекс 3) и V (индекс 5), направленные под одинаковым углом к нормали к поверхности: $\alpha_{3}=\alpha_{5}=\alpha$. Из условия материального баланса для атомов $\mathrm{Ga}$ имеем

$$
v_{3} \cos \alpha=N \frac{d}{d t}\left(\pi R^{2} L\right)+N \frac{d V_{d r o p}}{d t}+v_{r} .
$$

Здесь $v_{3} \cos \alpha-$ скорость осаждения Ga (эквивалентная скорость двумерного роста) из потока $v_{3}[\mathrm{~nm} / \mathrm{s}], N-$ поверхностная плотность цилиндрических ННК одинакового радиуса $R$ и длины $L, V_{d r o p}-$ объем капли на вершине ННК при ПЖК-росте, $v_{r}$ — поток $\mathrm{Ga}$, попадающий на подложку. При росте в регулярном квадратном массиве шага $P$ имеем $N=1 / P^{2}$. Толщина осаждения Ga за время $t$ равна $H=v_{3} \cos \alpha \cdot t$. Объем капли в дальнейшем предполагаем постоянным: $d V_{d r o p} / d t=0$, что требует определенного соотношения между контактным углом капли $\beta$ и радиусом $R$ в цилиндрической геометрии [14].

Начало асимптотической стадии соответствует обращению в нуль потока Gа на поверхность $\left(v_{r}=0\right)$, при этом ННК потребляют весь осаждаемый материал согласно (1). Это происходит при определенных значениях $H_{*}, R_{*}, L_{*}$, а также контактного угла капли $\beta_{*}$. Если непосредственно перед началом асимптотической стадии объем ННК увеличивался за счет прямого попадания $\mathrm{Ga}$ в каплю и на боковую поверхность ННК [8], из 

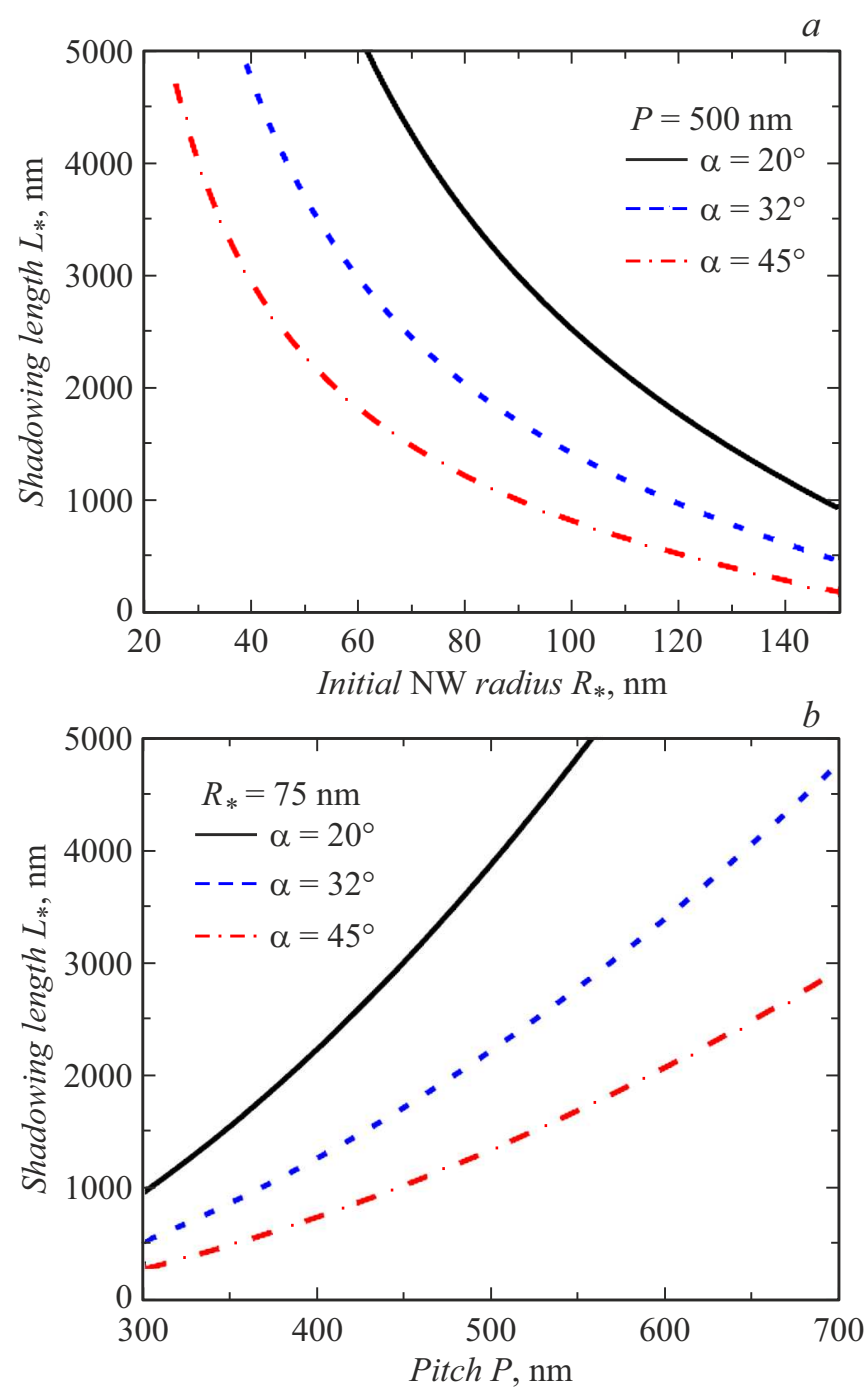

Рис. 1. Длина затенения, соответствующая полной блокировке поверхности подложки при МПЭ, в зависимости от радиуса ННК в начале асимптотической стадии при фиксированном $P=500 \mathrm{~nm}$ и трех различных $\alpha(a)$ и от расстояния между ННК при фиксированном $R_{*}=75 \mathrm{~nm}$ и тех же значениях $\alpha(b)$.

соотношения

$$
\frac{d}{d t}\left(\pi R^{2} L\right)=\left(\pi R^{2} \chi+2 R L \sin \alpha\right) v_{3}=\frac{v_{3} \cos \alpha}{N}
$$

при $R=R_{*}, L=L_{*}$ и $\chi=\chi_{*}$ получаем длину затенения в виде

$$
L_{*}=\frac{\operatorname{cotan} \alpha}{2 N R_{*}}-\frac{\pi R_{*} \chi_{*}}{2 \sin \alpha}
$$

В приведенных выражениях $\chi=\chi_{3}=\chi_{5}$ - геометрическая функция углов $\alpha$ и $\beta$, определяющая площадь сечения капли направленным потоком при МПЭ [15]. Согласно данным рис. 1 , длина $L_{*}$ убывает с ростом $R_{*}$ и возрастает с ростом расстояния между ННК $P$. Естественно, асимптотическая стадия наступает раньше при больших углах падения потока $\alpha$. В плотных массивах широких ННК асимптотическая стадия наступает факти- чески в самом начале роста (при длине ННК $\cong 300 \mathrm{~nm}$ для $R_{*}=75 \mathrm{~nm}, P=300 \mathrm{~nm}$ и $\left.\alpha=45^{\circ}\right)$.

Записывая (1) при $v_{r}=0$ в терминах $H$ в виде $d\left(R^{2} L\right) / d H=1 /(\pi N)$ и интегрируя с соответствующим начальным условием, получаем

$$
R=\left(\frac{h}{\pi N L}\right)^{1 / 2}, \quad h=H-H_{*}+\pi N R_{*}^{2} L_{*} .
$$

Закон удлинения ННК описывается известным выражением $[8,10,11]$ :

$$
\frac{d L}{d h}=\frac{\chi}{\cos \alpha}+\frac{2 \xi_{3} \lambda_{i n c} \tan \alpha}{\pi R}
$$

означающим, что длина ННК увеличивается за счет прямого попадания материала в каплю и диффузии фракции атомов $\mathrm{Ga} \xi_{3}$ с длины $\lambda_{i n c}$, в данном случае лимитированной встраиванием в ступени и боковым ростом ННК. Весь остальной материал затрачивается на боковой рост. Подстановка выражения (4) для $R$ в (5) приводит к уравнению Чини, которое исследовано в [16]. При автокаталитическом росте часто выполнено соотношение $d L / d t=\chi v_{5}$, означающее, что скорость удлинения ННК пропорциональна атомарному потоку элемента группы V (As) в каплю $[17,18]$. Переписав это выражение в виде

$$
\frac{d L}{d h}=\frac{\chi}{\cos \alpha} \frac{v_{5}}{v_{3}}
$$

и приравняв его к (5), получаем

$$
\chi=\frac{1}{v_{5} / v_{3}-1} \frac{2 \xi_{3} \lambda_{i n c} \sin \alpha}{\pi R} .
$$

Таким образом, постоянство объема капли Ga требует уменьшения $\chi$ (а значит, и контактного угла $\beta$ ) при росте радиуса ННК $R$.

Используя (7) в (5), получаем закон удлинения автокаталитического ННК

$$
\frac{d L}{d h}=c\left(\frac{L}{h}\right)^{1 / 2}, \quad c=\frac{1}{1-v_{3} / v_{5}} \frac{2 \xi_{3} \lambda_{i n c} \tan \alpha}{\pi}(\pi N)^{1 / 2}
$$

Его решение имеет вид

$$
L=\left[\sqrt{L_{*}}+c\left(\sqrt{h}-\sqrt{h_{*}}\right)\right]^{2}, \quad h_{*}=\pi N R_{*}^{2} L_{*} .
$$

Асимптотики при $h \gg h_{*}$

$$
L \rightarrow c^{2} h, \quad R \rightarrow R_{c}=\frac{1}{(\pi N)^{1 / 2} c}
$$

показывают, что длина ННК при больших временах пропорциональна толщине осажденного $\mathrm{Ga}$ и увеличивается с ростом коэффициента $c$. Радиус ННК стремится к стационарному значению $R_{c}$, которое уменьшается с ростом $c$. Поскольку значение $R_{N}=1 /(\pi N)^{1 / 2}$ соответствует слиянию ННК в сплошную пленку, рост 

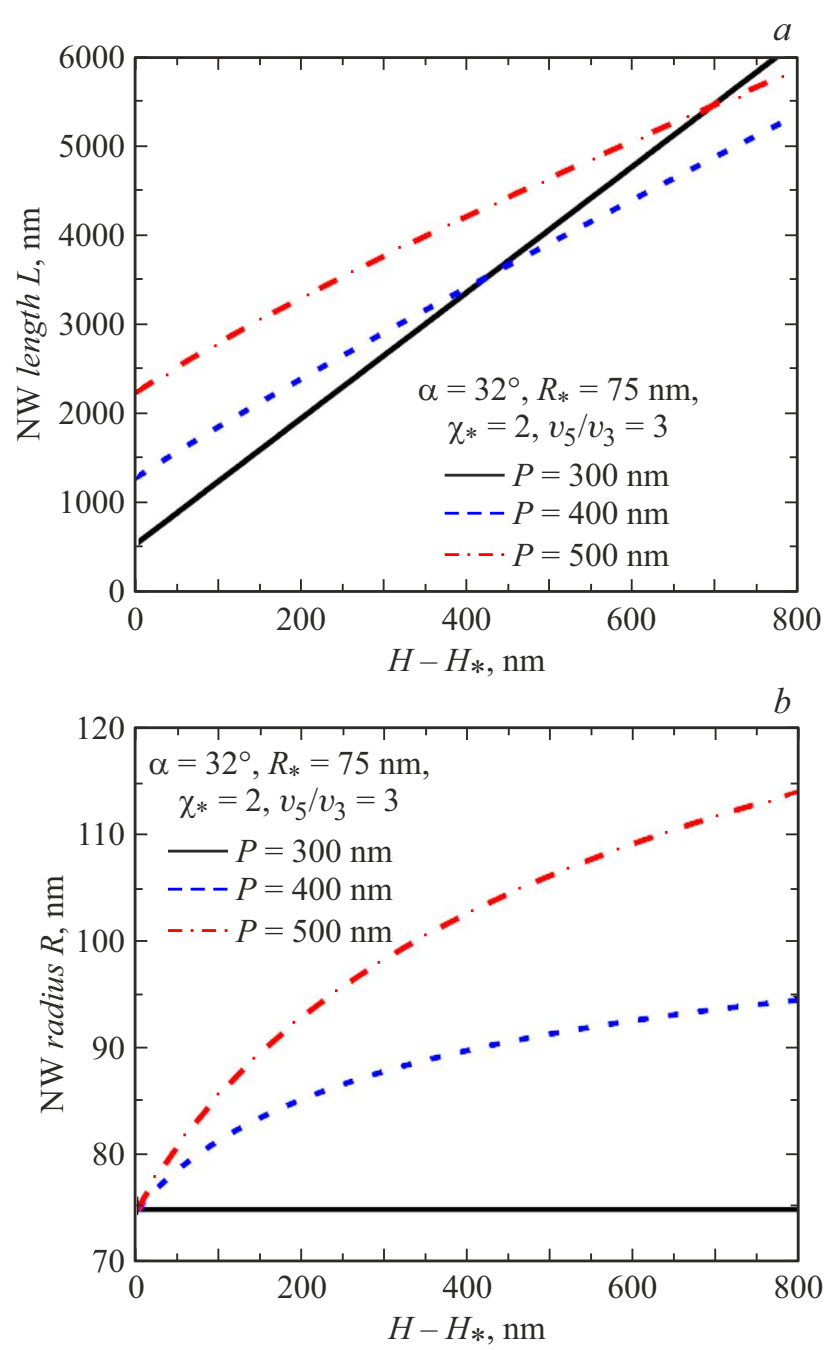

Рис. 2. Длина $(a)$ и радиус $(b)$ ННК в зависимости от толщины осаждения $\mathrm{Ga}$ c начала асимптотической стадии $H-H_{*}$ при параметрах, указанных на рисунке и в табл. 1, для трех различных $P$ и фиксированного соотношения атомарных потоков V/III $v_{5} / v_{3}=3$. При минимальном $P=300 \mathrm{~nm}$ асимптотическая стадия начинается при меньшей длине, однако в данном случае ННК растут только вверх и становятся длиннее других на позднем этапе роста. При увеличении расстояния между ННК усиливается радиальный рост, в результате чего зависимость длины ННК от $H-H_{*}$ (или от времени роста) медленнее линейной.

Таблица 1. Расчетные параметры кривых на рис. 2

\begin{tabular}{c|c|c|c|c}
\hline$P, \mathrm{~nm}$ & $L_{*}, \mathrm{~nm}$ & $c$ & $R_{c}, \mathrm{~nm}$ & $\chi_{c}$ \\
\hline 300 & 516 & 2.66 & 64 & 2 \\
400 & 1263 & 1.995 & 113 & 1.327 \\
500 & 2224 & 1.596 & 177 & 0.847
\end{tabular}

изолированных ННК при больших временах возможен только при $c>1$.

Разумеется, радиальный рост ННК может происходить только при $R_{*}<R_{c}$. В противном случае радиус ННК остается постоянным во времени, а его длина определяется обычным выражением

$$
L=L_{*}+\frac{\chi_{*}}{\cos \alpha} \frac{v_{5}}{v_{3}}\left(H-H_{*}\right) .
$$

Отметим также важные соотношения, следующие из (7), (8) и (10):

$$
c=\pi^{1 / 2} \chi_{*} \frac{v_{5}}{v_{3}} \frac{R_{*}}{P}, \chi=\chi_{*} \frac{R_{*}}{R}, R_{c}=\frac{v_{3}}{v_{5}} \frac{1}{\pi \chi_{*}} \frac{P^{2}}{R_{*}} .
$$
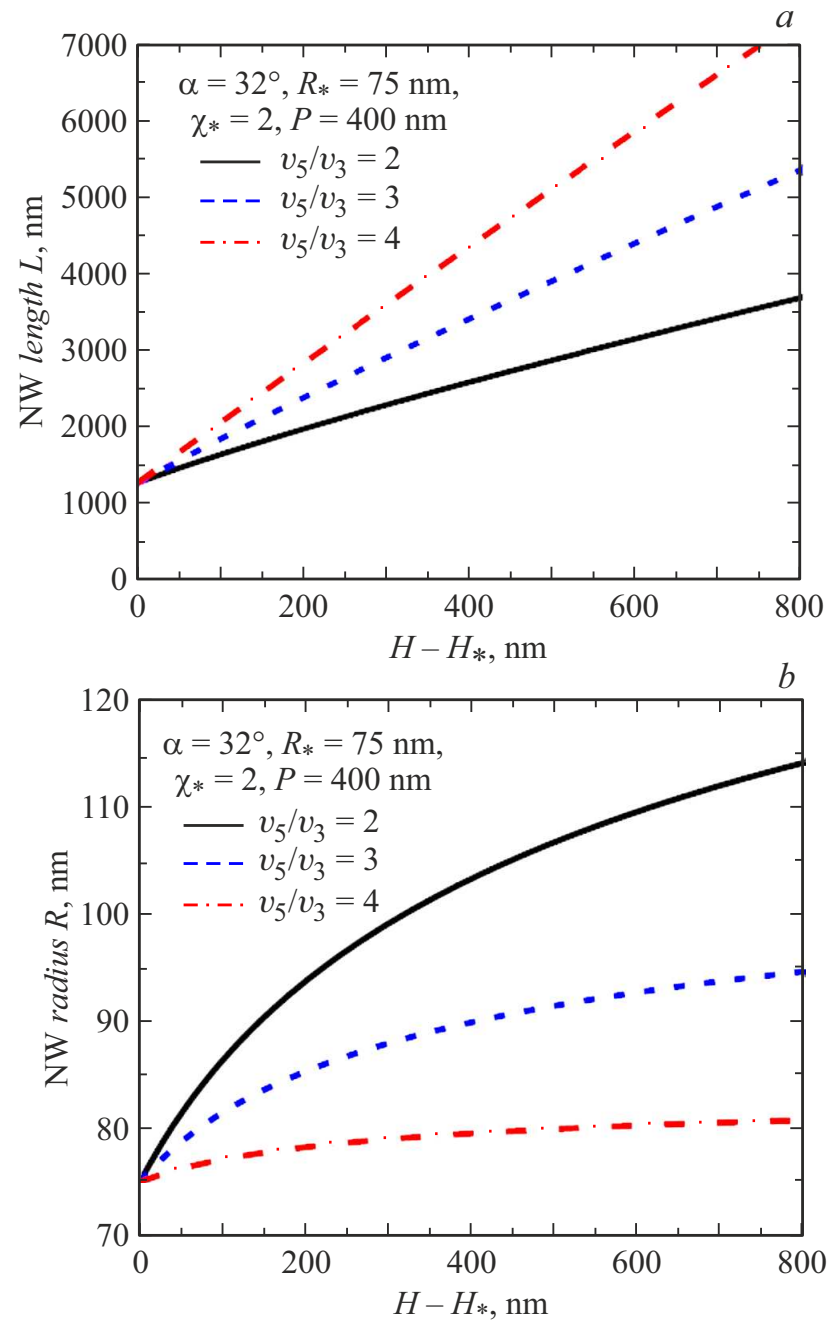

Рис. 3. Длина $(a)$ и радиус $(b)$ ННК в зависимости от толщины осаждения $\mathrm{Ga}$ при фиксированном $P=400 \mathrm{~nm}$ и трех различных соотношениях атомарных потоков V/III. Остальные параметры приведены в табл. 2. Осевой рост усиливается и радиальный рост ослабевает при увеличении соотношения потоков V/III.

Таблица 2. Расчетные параметры кривых на рис. 3

\begin{tabular}{c|l|c|c}
\hline$v_{5} / v_{3}$ & $c$ & $R_{c}, \mathrm{~nm}$ & $\chi_{c}$ \\
\hline 2 & 1.33 & 170 & 0.882 \\
3 & 1.995 & 113 & 1.327 \\
4 & 2.66 & 85 & 1.765
\end{tabular}


Они показывают непосредственно, что длина ННК увеличивается, а радиус уменьшается при увеличении соотношения потоков V/III $v_{5} / v_{3}$ и уменьшении расстояния между ННК $P$.

Формулы (4) и (9) для радиуса и длины автокаталитических ННК, а также соотношения (12) на асимптотической стадии роста являются основным результатом работы, проиллюстрированным на рис. 2 и 3. На рис. 2 приведены зависимости длины и радиуса ННК от толщины осажденного $\mathrm{Ga} H-H_{*}$ при фиксированном $v_{5} / v_{3}$ и различных $P$, а на рис. 3 - те же зависимости при фиксированном $P$ и различных $v_{5} / v_{3}$. Кривые на рис. 2 получены при $\alpha=32^{\circ}, \chi_{*}=2\left(\beta_{*}=135^{\circ}\right), R_{*}=75 \mathrm{~nm}$, $v_{5} / v_{3}=3$, остальные расчетные параметры представлены в табл. 1. Кривые на рис. 3 получены при $\alpha=32^{\circ}$, $\chi_{*}=2, R_{*}=75 \mathrm{~nm}, P=400 \mathrm{~nm}$, остальные параметры приведены в табл. 2. Заметим, что полученные асимптотические значения $\chi_{c}$ в обоих случаях соответствуют уменьшению контактного угла капли от $135^{\circ}$ до примерно $90^{\circ}$, что должно приводить к смене кристаллической фазы HНК GaAs с кубической на гексагональную и обратно в соответствии с [19].

В заключение отметим, что построенная модель допускает простые аналитические решения для длины и радиуса автокаталитических ННК на асимптотической стадии МПЭ-роста с учетом коллективного эффекта затенения. Результаты демонстрируют возможность управления морфологией ННК с помощью соотношения потоков V/III и расстояния между ННК.

\section{Финансирование работы}

Работа выполнена за счет гранта Российского научного фонда № 19-72-30004.

\section{Конфликт интересов}

Авторы заявляют, что у них нет конфликта интересов.

\section{Список литературы}

[1] A. Zhang, G. Zheng, C.M. Lieber, Nanowires: building blocks for nanoscience and nanotechnology (Springer, 2016).

[2] F. Glas, Phys. Rev. B, 74, 121302(R) (2006). DOI: 10.1103/PhysRevB.74.121302

[3] V.G. Dubrovskii, N.V. Sibirev, X. Zhang, R.A. Suris, Cryst. Growth Des., 10, 3949 (2010). DOI: 10.1021/cg100495b

[4] G.E. Cirlin, V.G. Dubrovskii, V.N. Petrov, N.K. Polyakov, N.P. Korneeva, V.N. Demidov, A.O. Golubok, S.A. Masalov, D.V. Kurochkin, O.M. Gorbenko, N.I. Komyak, V.M. Ustinov, A.Yu. Egorov, A.R. Kovsh, M.V. Maximov, A.F. Tsatusul'nikov, B.V. Volovik, A.E. Zhukov, P.S. Kop'ev, Zh.I. Alferov, N.N. Ledentsov, M. Grundmann, D. Bimberg, Semicond. Sci. Technol., 13, 1262 (1998). DOI: $10.1088 / 0268-1242 / 13 / 11 / 005$

[5] R.S. Wagner, W.C. Ellis, Appl. Phys. Lett., 4, 89 (1964). DOI: $10.1063 / 1.1753975$
[6] C. Colombo, D. Spirkoska, M. Frimmer, G. Abstreiter, A. Fontcuberta i Morral, Phys. Rev. B, 77, 155326 (2008). DOI: 10.1103/PhysRevB.77.155326

[7] S. Hertenberger, D. Rudolph, M. Bichler, J.J. Finley, G. Abstreiter, G. Koblmüller, J. Appl. Phys., 108, 114316 (2010). DOI: $10.1063 / 1.3525610$

[8] V.G. Dubrovskii, F. Glas, in: Fundamental properties of semiconductor nanowires, ed by N. Fukata, R. Rurali (Springer, 2020), p. 3-107.

DOI: 10.1007/978-981-15-9050-4_1

[9] N.V. Sibirev, M. Tchernycheva, M.A. Timofeeva, J.C. Harmand, G.E. Cirlin, V.G. Dubrovskii, J. Appl. Phys., 111, 104317 (2012). DOI: 10.1063/1.4718434

[10] Г.Э. Цырлин, В.Г. Дубровский, Н.В. Сибирев, И.П. Сошников, Ю.Б. Самсоненко, А.А. Тонких, В.М. Устинов, ФТП, 39 (5), 587 (2005). [G.E. Cirlin, V.G. Dubrovskii, N.V. Sibirev, I.P. Soshnikov, Y.B. Samsonenko, A.A. Tonkikh, V.M. Ustinov, Semiconductors, 39 (5), 557 (2005). DOI: $10.1134 / 1.1923565]$.

[11] M.C. Plante, R.R. LaPierre, J. Appl. Phys., 105, 114304 (2009). DOI: 10.1063/1.3131676

[12] S.J. Gibson, R.R. LaPierre, Nanotechnology, 25, 415304 (2014). DOI: $10.1088 / 0957-4484 / 25 / 41 / 415304$

[13] F. Oehler, A. Cattoni, A. Scaccabarozzi, J. Patriarche, F. Glas, J.C. Harmand, Nano Lett., 18, 701 (2018). DOI: $10.1021 /$ acs.nanolett.7b03695

[14] V.G. Dubrovskii, I.P. Soshnikov, G.E. Cirlin, A.A. Tonkikh, Yu.B. Samsonenko, N.V. Sibirev, V.M. Ustinov, Phys. Status Solidi B, 241, R30 (2004). DOI: 10.1002/pssb.200409042

[15] F. Glas, Phys. Status Solidi B, 247, 254 (2010). DOI: $10.1002 /$ pssb.200945456

[16] V.G. Dubrovskii, Nanomaterials, 11, 2378 (2021). DOI: $10.3390 /$ nano11092378

[17] F. Glas, M.R. Ramdani, G. Patriarche, J.C. Harmand, Phys. Rev. B, 88, 195304 (2013). DOI: $10.1103 /$ PhysRevB.88.195304

[18] J. Tersoff, Nano Lett., 15, 6609 (2015). DOI: $10.1021 /$ acs.nanolett.5b02386

[19] V.G. Dubrovskii, Cryst. Growth Des., 17, 2544 (2017). DOI: 10.1021/acs.cgd.7b00076 